\title{
Cytotoxicity of Chlorinated Hydrocarbons and Lipid Peroxidation in Isolated Rat Hepatocytes
}

\author{
Toshinide Suzuki, Haruyo Sasaki and Hideo Isono \\ Faculty of Phurmaceutical Sciences, Teikyo University, 1091-1 \\ Sagamiko-machi, Tsukui-gun, Kanagawa 199-01, Japan
}

\section{Introduction}

With the recent attention paid to the toxicities of chlorinated hydrocarbons, ten varieties were subjected to environmental standards in Japan in 1993. Peroxidation of biological membrane lipid is suggested to be an integral part of cell damage and of many toxic processes. It is presumed that many chlorinated hydrocarbons induce membrane lipid peroxidation and thus cytotoxicity. In this study, we used a sensitive and selective assay for phosphatidylcholine hydroperoxide $(\mathrm{PCOOH})$ and phosphatidylethanolamine hydroperoxide $(\mathrm{PEOOH})$ using high-performance liquid chromatography combined with chemiluminescence detection (CL-HPLC), and investigated the relationship between cytotoxicity and phospholipid hydroperoxidation in rat hepatocytes by chlorinated hydrocarbons. ${ }^{1)}$

\section{Methods}

Hepatocytes were prepared from male Wistar rat (10 weeks of age, $250 \mathrm{~g})$. Chlorinated hydrocarbons (dichloromethane, chloroform, carbon tetrachloride, 1,2-dichloroethane, 1,1,1trichloroethane, 1,1,2-trichloroethane, 1,1dichloroethylene, cis-1,2-dichloroethylene, trichloroethylene, tetrachloroethylene and 1,3dichloropropene) were each diluted in dimethyl sulfoxide, and were dissolved in Eagle's MEM to give a final concentration of $10 \mathrm{~mm}$. Two hours after the incubation, the medium lactate dehydrogenase (LDH) activity and the cellular protein amount attached to culture dishes were measured as an index of cytotoxicity. The CL-HPLC system and experimental conditions for measuring phospholipid hydroperoxides were the same as those previously described by Miyazawa et al. Thiobarbituric acid reactive substance (TBA-RS) was measured by the method of Ohkawa et al.

\section{Results and Discussion}

Severe cytotoxicity (increase in activity of medium LDH (6.3-10.5 times) and decrease in amount of attached protein $(22-53 \%)$ ) were observed in the presence of carbon tetrachloride, 1,1,1-trichloroethane, tetrachloroethylene and 1,3-dichloropropene at a concentration of $10 \mathrm{~mm}$. The cellular $\mathrm{PCOOH}$ level was remarkably increased after incubation with 1,3-dichloropropene (8.5 times), 1,1,1-trichloroethane (6.2 times), carbon tetrachloride (5.3 times) and tetrachloroethylene (4.2 times) as compared with the control cultures, respectively. The $\mathrm{PCOOH}$ levels were not increased by incubation with the other compounds, which did not show cytotoxicity. The same tendency as for $\mathrm{PCOOH}$ was observed with $\mathrm{PEOOH}$. The concentration of TBA-RS was increased by incubation with carbon tetrachloride (1.7 times), 1,1,1-trichloroethane (1.6 times), tetrachloroethylene (1.6 times) and 1,3dichloropropene (1.4 times), respectively. To evaluate the relationship between cellular lipid hydroperoxidation and such toxic substances as drugs, heavy metals and environmental pollutants, the direct detection of lipid hydroperoxide is very important.

In conclusion, severe hepatocellular toxicity was confirmed with exposure to carbon tetrachloride, 1,1,1-trichloroethane, tetrachloroethylene and 1,3-dichloropropene, accompanied by a remarkable increase in generation of phospholipid hydroperoxide. The peroxidative degradation of cellular membrane phospholipids may be one of the important causes of cytotoxicity induced by some chlorinated hydrocarbons.

\section{Reference}

1) T. Suzuki, K. Nezu, H. Sasaki, T. Miyazawa and H. Isono, Biol. Pharm. Bull., 17, 82 (1994). 